Forschende

Komplementärmedizin und

Klassische Naturheilkunde
Kongressbericht · Congress Report

Forsch Komplementärmed Klass Naturheilkd 2001;8:305-306

\section{Symposium zur Ratio und Plausibilität in der Naturheilkunde}

\section{«Naturheilkundliche Konzepte in der Schmerztherapie»}

Seit 1999 veranstaltet das Institut für Physiotherapie am Klinikum der Friedrich-Schiller-Universität in Jena jährlich ein Symposium «Zur Ratio und Plausibilität in der Naturheilkunde». Die wissenschaftliche Leitung und Organisation liegt in den Händen von Frau Prof. Dr. med. C. Uhlemann. Die erste Veranstaltung am 18.12.1999 thematisierte die Naturheilverfahren als integrativen Bestandteil der «Schulmedizin». Nach einer lebhaften Diskussion wurde allgemein zugestimmt: In dem bewährten System der modernen Schul- oder Hochschulmedizin gründen auch die tradierten (klassischen) Naturheilverfahren auf einer gesunden wissenschaftlichen Basis und einer zunehmend erkennbaren Ratio ihrer Indikationen und therapeutischen Strategien. Mit neuen Ergebnissen zur Pathogenese und Nosologie gewinnen ihre Therapien zunehmend an Plausibilität, auch hier partizipiert die Naturheilkunde an den Fortschritten moderner klinischer Grundlagenforschung.

Die zweite Veranstaltung am 09.12.2000 wandte sich einem praktisch relevanten klinischen Thema, der Schmerztherapie, zu.

In der Algesiologie haben rasante Entwicklungen stattgefunden, die auch für die Naturheilkunde ein neues Verständnis eröffnen. Ist der medizinische Zeitgeist in Fragen der «Schmerzforschung» vornehmlich molekularbiologisch und elektrophysiologisch ausgerichtet, so bekommt er durch die «Rationale» der klassischen Naturheilverfahren eine notwendige Ergänzung.

Die Phänomenologie, Physiologie und Pathologie des Schmerzes geben Anlass, das Menschenbild in der Medizin mit Human- und Biowissenschaft gleichzeitig auszubauen. Nie sonst werden Psyche und Soma derart verbunden und gleichzeitig untersucht.

Manche Autoren entwickeln am Beispiel des Schmerzes einen neuen Monismus in der Medizin.

Erst mit einer psychosomatischen Identität - nicht nur mit einer Parallelität - wäre der Mensch wieder «ganz». Ganz im Sinne von «heil» oder wieder gesund.

Wie selten sonst in der Medizin wird im Umgang mit Schmerz eine zweite Art ganzheitlicher Betrachtung gefordert. Schmerz spielt sich regelhaft auf allen Ebenen der Entität Mensch gleichzeitig ab. Mit dieser Ganzheit ist mehr gemeint als das heute favorisierte bio-psycho-soziale Modell des Menschen.

In der jetzt referierten Veranstaltung wurden eingangs moderne Aspekte zur «Pathophysiologie des Schmerzes» vorgetragen und interpretiert (H.-G. Schaible, Jena); von grossem Interesse waren neue Ergebnisse zur peripheren und zentralen Nozizeption. Phänomene wie die periphere und zentrale Sensibilisierung und mögliche Mechanismen der Schmerzchronifizierung wurden akzentuiert. Das «Sichtbarmachen des Schmerzes», d.h. die Erfassung sensorischer und emotionalmotivationaler Prozesse durch Darstellung der neuronalen Aktivität sowie der zerebralen Stoffwechselvorgänge wurde als modernes Ergebnis der Schmerzforschung vorgestellt.

Danach interpretierte M. Bühring (Berlin) als Vertreter der klassischen Naturheilverfahren den Schmerz mit seinen Facetten als ein Erlebnis und als ein Leiden auf allen Ebenen der menschlichen Existenz. Dabei wurden auch Charakteristika der naturheilkundlichen Philosophie abgehandelt, die bionomes Handeln, nosologische und konstitutionelle Bezüge bzw. Betrachtungen genauso einbezieht wie Selbstheilungskräfte und metaphorische und transzendentale Deutungen und Bedeutungen.

Anschliessend wurden fünf Vorurteile gegenüber der medikamentösen Schmerztherapie seitens des anästhesiologischen Algesiologen (W. Meissner, Jena) ausgeräumt:

«Opiate machen süchtig»

- nein, bei adäquater Dosis;

«Analgetika benebeln»

- nein, bei adäquater Dosis;

«Analgetika fördern Passivität

des Patienten»

$-j a ;$

«Analgetika sind organtoxisch» - NSAR: ja, Opiate: nein;

"Phytotherapeutika sind besser als synthetische Analgetika»

\section{- Datenlage noch nicht aus- reichend; Ansätze, um deren Effizienz zu zeigen, existieren.}

Defizite im Wissen um die adäquaten Schmerzmittel und deren Dosierung sowie Galenik, insbesondere bei den Morphinen, für die entsprechenden Indikationen sind noch vorhanden und erklären Berührungsängste einiger Ärzte bei Verordnungen, z.B. von Opiaten.

C. Uhlemann (Jena) war es ein Anliegen, in dieser Veranstaltung über Naturheilkunde auch die Physikalische Therapie als eine zuverlässige und teilweise belegte Säule in der Behandlung von Schmerzen darzustellen. Einerseits referierte sie Hypothesen zur Wirkungsphysiologie mechanischer, thermischer und elektrischer Reize, andererseits trug sie Ergebnisse von Therapiestudien zu therapeutischen Effekten dieser physikali-

\section{KARGER}

Fax +49 7614520714

() 2001 S. Karger GmbH, Freiburg

E-mail Information@Karger.de www.karger.com/journals/fkm

www.karger.com 
schen Reize bei ausgewählten Indikationen (Fibromyalgiesyndrom, Enthesopathie, Osteoporose, Arthritis) vor. Letztlich sind die Optionen einer Physikalischen Therapie als Schmerztherapie in der Beeinflussbarkeit von Symptomen/Syndromen, von gestörten physiologischen Regelsystemen und von einer veränderten «bio-psycho-sozialen Befindlichkeit» zu sehen.

Der aktuelle Stand der Forschung zur Akupunktur, einer probaten und praktisch relevanten Option in der Schmerztherapie, als Reflex- und Regulationstherapie wurde mit Bezugnahme zu Problemen wissenschaftlicher Studiengestaltung entsprechend einer Evidence-Based Medicine dargestellt (D. Irnich, München). Diese Probleme beinhalten einmal eine Placebokontrolle und die Verblindung des Akupunkteurs sowie zum anderen das individuelle Therapieschema und die Objektivierbarkeit von Therapieeffekten. Die Evidenz für die Wirksamkeit der Akupunktur ist bei ausgewählten Indikationen erbracht, wozu der Kreuzschmerz, die Arthrose und der Kopfschmerz zählen. Auch wurden Aussagen zur Akupunktur als Gegenstand berufspolitischer Ränkespiele gemacht.

Die Neuraltherapie als Reflextherapie mit vergleichbarem Duktus zu anderen reflextherapeutischen Massnahmen (Akupunktur, Manuelle Therapie) wurde am Beispiel der Kopfschmerzintervention entwickelt (R. Wander, Elsterberg). Hypothetische Spekulationen über die Relevanz der Verschaltung parasympathischer Kerne (sakral und zervikal) gaben Anlass zur Diskussion.

Therapeutische Wirksamkeiten von Phytopharmaka in der Schmerztherapie vornehmlich rheumatischer Krankheiten und der Neuropathie wurden an Hand von Studienergebnissen als effektive adjuvante Therapie vorgestellt (B. Uehleke, Rostock). Thematisiert wurden positiv monografierte Phytoanalgetika wie der Weidenrindenextrakt, die Teufelskralle, die Pestwurz, die Brennessel und das Capsaicin.

Abschliessend wurde Gesichertes und Plausibles zur Ernährungstherapie in der Schmerztherapie referiert (R. Stange,
Berlin). Dabei war der Darm als «Markt» pathogenetischer und therapeutischer Spekulationen zentraler Sachverhalt. Der Darm als grösstes Immunsystem generiert mit seiner veränderten Mucosabarriere zahlreiche sogenannte chronische Befindlichkeitsstörungen und spielt bei chronisch entzündlichem Geschehen (z.B. CED, RA, SPA, Psoriasisarthritis, Asthma) eine wesentliche Rolle. Die therapeutische Relevanz einer adäquaten Ernährung ist evident.

Jena ist wie kein anderer Ort berufen, Gedanken zur Ganzheit wieder aufzugreifen. In C.W. Hufeland kumulierte in dieser Stadt noch einmal die ausklingende Naturphilosophie, Jena war Zentrum und Höhepunkt der romantischen Medizin, später haben E. Klein und J. Grober - in unglücklicher persönlicher Verstrickung - Naturheilkunde zu einer überzeugenden Physiotherapie geführt.

Für jeden Arzt ist die Behandlung von Schmerzen eine elementare Aufgabe und Herausforderung zugleich. Dabei ist der Molekularbiologe genauso gefordert wie der Naturheilkundler. Nur sollte jeder die Theorie des anderen kennen und im symbiotischen Gemeingang für den Patienten benefitabel nutzen.

Zweck dieser Veranstaltung ist es, den Dialog zwischen Vertretern der dominierenden sogenannten Schulmedizin und solchen der klassischen Naturheilverfahren zu fördern. Diese Aufforderung wurde von universitären Kollegen genauso angenommen wie von niedergelassenen. Dass Studenten gleichsam wie Patientenvertreter ebenso wissensdurstige Zuhörer waren, zeugt von der Notwendigkeit einer «ganzheitlichen» Darstellung der Phänomene des Lebens an sich.

Die Fortsetzung dieser Veranstaltungsreihe findet am 08.12.2001 wiederum in Jena statt. Diesmal werden «Betrachtungen zum Fibromyalgiesyndrom aus sogenannter schulmedizinischer und naturheilkundlicher Sicht» angestellt.

C. Uhlemann, Jena

Funktionsbereichsleiterin des Instituts für Physiotherapie, Friedrich-Schiller-Universität, Jena 